\title{
Editorial
}

\section{Advanced Engine Flows and Combustion}

\author{
Zhijun Peng, ${ }^{1}$ Thanos Megaritis, ${ }^{2}$ Chih-Jen Sung, ${ }^{3}$ Minoru Yaga, \\ Paul Hellier, ${ }^{5}$ and Guohong Tian ${ }^{6}$
}

${ }^{1}$ University of Bedfordshire, Luton, UK

${ }^{2}$ Brunel University London, Uxbridge, UK

${ }^{3}$ University of Connecticut, Storrs, CT, USA

${ }^{4}$ University of the Ryukyus, Okinawa, Japan

${ }^{5}$ University College London, London, UK

${ }^{6}$ University of Surrey, Guildford, UK

Correspondence should be addressed to Zhijun Peng; jun.peng@beds.ac.uk

Received 12 July 2017; Accepted 12 July 2017; Published 7 August 2017

Copyright (c) 2017 Zhijun Peng et al. This is an open access article distributed under the Creative Commons Attribution License, which permits unrestricted use, distribution, and reproduction in any medium, provided the original work is properly cited.

The transport sector accounts for a significant part of carbon emissions worldwide, and so the need to mitigate the greenhouse effect of $\mathrm{CO}_{2}$ from fossil fuel combustion and to reduce vehicle exhaust emissions has been the primary driver for developing cleaner and more efficient vehicle powertrains and environmentally friendly fuels. As alternatives to combustion engines have yet to overcome technical challenges to attain significant utilisation in the transport sector, piston-driven internal combustion engines and gas turbine aeroengines remain very attractive powertrain options due to their high thermal efficiency. Meanwhile, since the introduction of various emissions standards, which have forced the employment of various aftertreatment systems, the evolution of combustion process has been significant. Advanced combustion strategies have attempted to find inchamber approaches either to meet these emission standards fully and thus avoid the need to use aftertreatment or, at the very least, to lower the performance demands required from aftertreatment systems thus reducing their cost and complexity. While the main focus of combustion system development has been recently to lower emissions of $\mathrm{CO}_{2}$, there is also significant interest to lower nitric oxides (NOx) and particulate matter (PM) emissions and other harmful emissions.

For piston-driven internal combustion engines, the most recent technology to have been successfully commercialised is Gasoline Direct Injection (GDI). GDI technology enables the air-fuel charge to be appropriately stratified so that ultralean burn combustion for improved fuel efficiency and reduced emissions can be achieved. As fuel is injected at the latter stages of the compression stroke, combustion takes place in a cavity on the piston's surface which has normally a toroidal shape and is either placed in the centre (for a central injector) or displaced to one side of the piston that is closer to the injector position. The cavity creates swirl or tumble effects so that the small amount of air-fuel mixture is optimally placed near the spark plug. This stratified charge is surrounded mostly by air and residual gases, thereby keeping the fuel and the flame away from the cylinder walls. Those requirements demonstrate that it is critical for GDI engines to organise appropriate air motion around the cavity area to provide suitable air-fuel ratios for spark ignition and in the whole cylinder for necessary charge stratification.

Lean burn with decreased combustion temperature in GDI engines can provide low emissions and low heat losses, leading to improved efficiency. However, a new devastating knock named superknock, which is much different from traditional gasoline engine knock combustion and whose instantaneous in-cylinder pressure can reach above 200 bars, has become a main obstacle for increasing GDI engine power density. Researchers are actively engaged in researching superknock, but the mechanism by which it is generated and practical control strategy are still not fully clarified. A mainstream view is that, in a superknock cycle, the occurrence of 
preignition is related to the dilution of the cylinder oil which may be caused by spray impingement on cylinder wall for turbocharged GDI engines. The viscosity of the diluted oil decreases with high temperature in combustion chamber, and the diluted oil can easily escape into the combustion chamber from the cylinder wall. Since oil typically has a high cetane number and its autoignition temperature is much lower than that of gasoline, the preignition of mixtures with oil in the late compression stroke occurs before spark ignition timing, inducing superknock. More researches are still needed for investigating injection impingement, interaction between fuel injection and oil film, and oil droplet's characteristics in combustion chambers of GDI engines.

Unlike GDI which can directly dominate engine combustion systems as a single combustion technology, Low Temperature Combustion (LTC) has mainly infiltrated into various combustion systems as a new combustion mode to reduce combustion temperature and improve combustion efficiency. LTC technology, which can work for both gasoline and diesel engines, has been widely studied in recent years due to advantages of low NOx emission and simultaneously high efficiency. Early works on piston engines with LTC demonstrated that engine-out NOx and PM emissions could be lowered to about $1-10 \%$ of conventional gasoline and diesel engine technologies. To implement LTC in diesel or gasoline engines, the control of ignition timing, which cannot be directly regulated as is the case in conventional internal combustion engines with fuel injection or spark ignition, must be dealt with. Because load is dependent on both the combustion phasing and the amount of reactant species present in the cylinder, both load and combustion phasing are therefore coupled and dependent on in-cylinder species concentrations, temperature, and pressure. Starting LTC with the previous cycle, a certain amount of residual gas should be trapped, while both air and EGR (Exhaust Gas Recirculation) in the correct proportions and at the right temperature must be added. Fuel is added in such a way that it evaporates and is then dispersed to support the premixed mode of combustion. Existing research results have suggested that VVA/VVT (Variable Valve Actuation/Variable Valve Timing) are effective for operating LTC mode, particularly for practically transient operating conditions. When VVA/VVT are employed, their effects on in-cylinder flows and the subsequent influences of these on air-mixing and ignition process become complicated and must be comprehensively examined for utilising the maximum benefit of LTC technology.

In terms of gas turbine aircraft engines, for achieving low emissions, low specific fuel consumption, and low cost of manufacture/maintenance, it is a great challenge for combustor design to reduce NOx emission without negative effects on other performances of the combustor. Recent approaches for NOx reduction had been presented including fuel staging, some applied combustors such as dual annular combustor, direct injection and Rich burn/Quick quench/Lean burn (RQL) and Lean Premixed and Prevaporized (LPP) combustion, and so on. For instance, premixer-prevaporizer combustor, which is a key technology for NOx emission reduction, has been presented in the form of a number of different designs. One design is to use an air assisted pressure swirl atomizer to atomize fuel, while a circular premixer was fitted at the end of the atomizer. Then swirl, which is necessary for flame stabilization, is created by axially oriented swirl vanes at the end of the premixer. Another LPP combustor design is to use a premixer-prevaporizer at main stage with a conventional injection used in pilot stage, and then it becomes a dual annular combustor. The LPP combustor structure for this design consisted of a swirl cup injection with a lengthened sleeve to form a LPP tube. Fuel-air mixing/preparation devices in aeroengines not only directly influence ignition process, lean blow-out, combustion efficiency, and fuel consumption but also have significant effects on emissions and exit temperature distributions. Swirl cups, whose main components normally include primary-swirler, Venturi, secondary-swirler, flare, and fuel nozzle, have been applied in many combustion systems. These technologies are currently under intensive investigation, including studies of flow fields, aerodynamics, spray structure and atomization, air-fuel mixing, combustion processes, and emissions.

Combustion processes in gas turbine engines are very sensitive to fluctuations of pressure, density, and temperature of the environment. Even slow changes of those quantities will affect the energy released according to rules that can be deduced from the behaviour for steady combustion. Combustion instabilities normally occur in frequency ranges such that genuine dynamical behaviour is significant. Any fluctuation in burning produces local changes in the properties of the flow. Then those fluctuations propagate in the medium and join the global unsteady field in the chamber. The dynamical response of the medium converts the local fluctuations into global behaviour. For various combustion systems and new combustion technologies, combustion instability is always one of the main challenges and significant attention must be paid to these details in flows and combustion.

The International Energy Agency estimates that biofuels can grow to as much as $30 \%$ of the world's road transport fuel mix by 2050 . Such fuels will include biodiesel and synthetic diesel fuels from waste sources via processes such as Fisher Tropsch. In the same time-frame, alcohols such as bioethanol produced from nonfood sources with reduced production costs and low $\mathrm{CO}_{2}$ emissions have been proposed as alternative fuels for direct blending with diesel, biodiesel, or synthetic diesel. According to Shell, one of main suppliers of biofuel, ethanol made from Brazilian sugar cane produces around $70 \%$ lower $\mathrm{CO}_{2}$ emissions from production to use compared to gasoline. Therefore, following ethanol-gasoline blends and direct biodiesel, potentials of ethanol-diesel blends (e-diesel) as alternative fuel for low carbon advanced diesel engines have been studied by some researchers. It is likely that an increasingly diverse range of alternative biofuels, of varying molecular structure, will be utilised in the future as further emphasis is placed on sustainable production routes that result in reduced emissions of greenhouse gases (GHG) over the fuel production and usage lifecycle. These will likely contain molecules such as furans or terpenes from sources such as lignocellulosic biomass or genetically engineered microorganisms, and much research is still required so as to fully understand the impact of possible 
future fuels such as these on ignition characteristics and the production of toxic pollutants.

In addition, it has been noticed that microexplosion may be exceedingly possible to occur during spray atomisation and combustion of fuel blends with differences of physical properties among the different fuels in the mixture. As one possible key phenomenon the understanding of which is necessary for using multicomponent fuels, microexplosion of a miscible multicomponent fuel droplet is due to the difference of volatility and boiling point among the different components. For an immiscible multicomponent fuel droplet (emulsion droplet as routinely termed), the likelihood of microexplosion will considerably increase if the lowerboiling-point component cannot dissolve in the mixture and disperse as microdroplets inside the fuel droplet, such as in the case of e-diesel as the volume fraction of bioethanol increases. Studies have shown that water emulsified in fossil fuels used in all combustion systems can lead to reductions in the adiabatic flame temperature, resulting in measurable reductions of $\mathrm{NO}_{x}$ emissions. Because of the difference in the evaporation rates of liquid diesel and water, the water molecules reached their superheated stage faster than the diesel, creating vapour expansion breakup. It is at this stage that the two phenomena, microexplosion and puffing, exist. Microexplosion is the rapid breakup of the emulsion droplets into smaller droplets, while, in puffing, water leaves the droplets in a very fine mist. These microexplosions result in a fast breakdown or secondary atomization of the fuel droplet, which, in turn, causes rapid fuel evaporation and, hence, leads to an improved air-fuel mixing. More studies on the preparation of emulsified water-fuel mixtures and multicomponent fuels and microprocessing of microexplosion and puffing under different operating conditions are necessary for applying the technology on practical engines.

As relevant researches have been moved to more detailed microresolutions including spatial and temporal ones during more complicated processes, various advanced optical diagnostics have been widely developed and applied for examining flows and combustion processes. Since flow fields, including their velocity distributions, temperature distribution, and species distributions, in both piston engines and gas turbines are at high level turbulent scales, optical particularly laser based diagnostic tools which bring no disturbance to the flow and reaction field are so helpful to measure and obtain information during detailed transient flow or combustion process inside combustion chambers. In addition to those more generally popular laser diagnostics such as nonspectroscopic PIV (Particle Image Velocimetry), LDA (Laser Doppler Velocimetry), PDA (Phase Doppler Anemometry), spectroscopic LIF (Laser Induced Fluorescence), and LII (Laser Induced incandescence), using X-ray Phase Contrast Imaging for investigating cavitation and gas ingestion in practical diesel injectors, DIH (Digital In-Line Holography) for microparticle characterisation, RS (Raman Scattering) for different species concentrations simultaneously, CARS (Coherent anti-Stokes Raman spectroscopy) for combustion species and temperature, TDLAS (Tuneable Diode Laser Absorption Spectroscopy) for species concentrations, and
MTV (Molecular Tagging Velocimetry) for velocity measurement in supersonic combustion has been recently reported.

Meanwhile, numerical simulation methods have also achieved significant development in recent years. Large Eddy Simulation (LES), in which the larger scales of turbulence are directly solved, where smaller scales are modelled using the subgrid models due to their isotropic characteristics, has been applied to engine combustion simulation in the last two decades. As LES is able to obtain more information of the turbulent flow field compared to RANS (ReynoldsAveraged Navier-Stokes) model, whilst less computational requirement is needed relative to DNS (Direct Numerical Simulation), LES has been expected to be the main way to model engine combustion.

Though the computational cost of DNS is very high and the computational resources required by a DNS for general combustion system will exceed the capacity of the most powerful computers currently available, it is still a useful tool in fundamental research in turbulent flows and combustion. Relevant "numerical experiments" by using 1D, 2D, and 3D DNS to complete simulation for premixed combustion have extracted required information which is difficult or impossible to obtain in the laboratory. This can allow a better understanding of the physics of turbulent flows and combustion. Meanwhile, DNS simulations are useful in the development of turbulence models for practical applications, such as subgrid scale models for LES and models for methods that solve RANS. This is done by means of "a priori" tests, in which the input data for the model is taken from a DNS simulation, or by "a posteriori" tests, in which the results produced by the model are compared with those obtained by DNS. DNS models and simulation methods will be definitely main research areas in the near future for turbulent flows and combustion, and it should be gradually able to provide significant help for new combustion system developments.

This edition is aiming to collect high-quality research articles and reviews that seek to address recent development on flow characteristics, air-fuel mixing, ignition and combustion processes, and emission reduction. Relevant prospects on opportunities and challenges for alternative fuels and novel combustion concepts have also been included with researches by both experimental works and numerical simulations.

Zhijun Peng
Thanos Megaritis
Chih-Jen Sung
Minoru Yaga
Paul Hellier
Guohong Tian




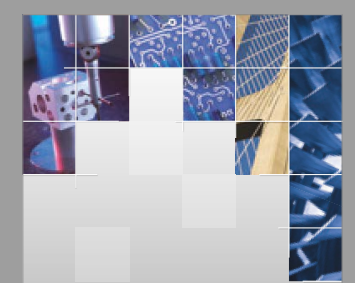

\section{Enfincering}
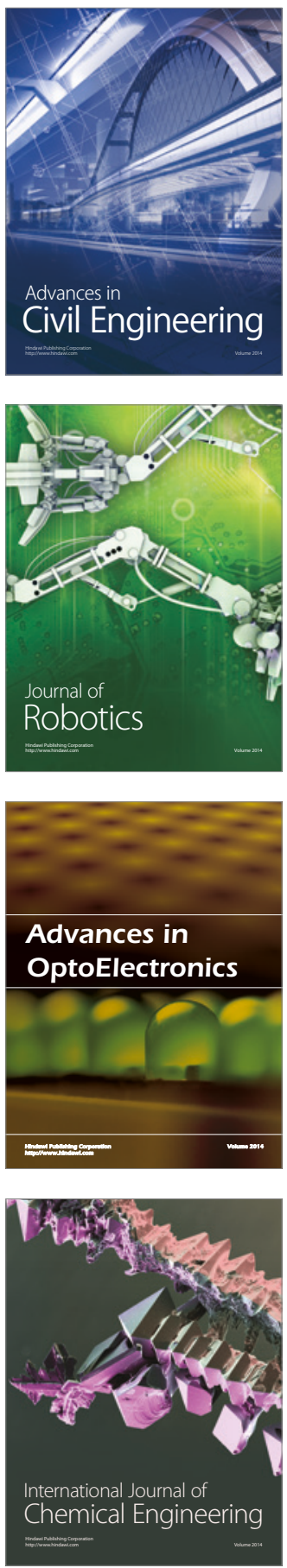

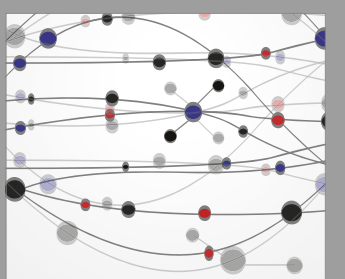

The Scientific World Journal

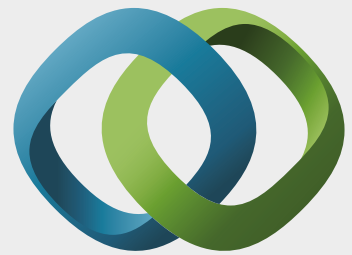

\section{Hindawi}

Submit your manuscripts at

https://www.hindawi.com
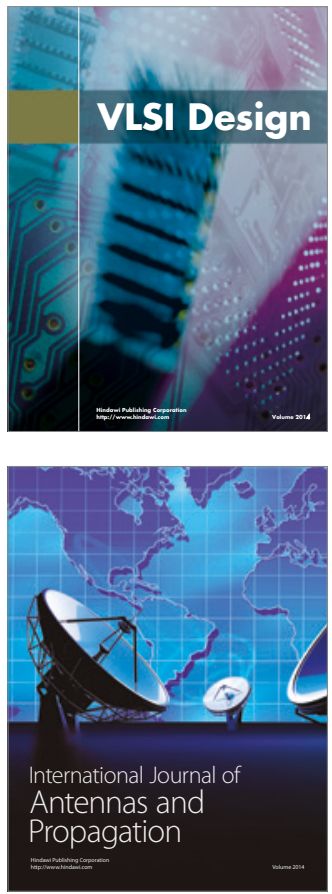

\section{Rotating}

Machinery
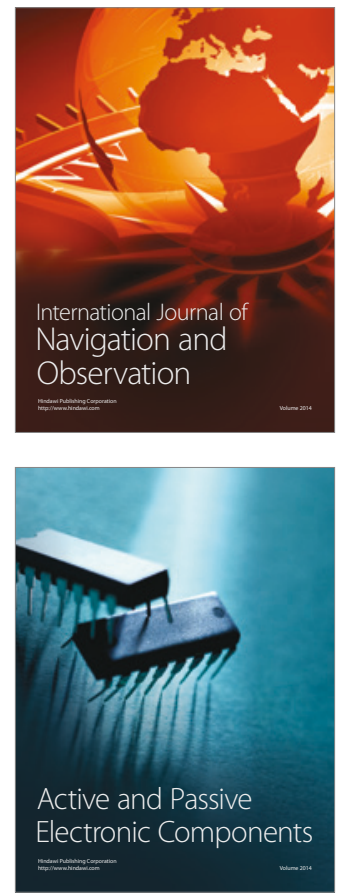
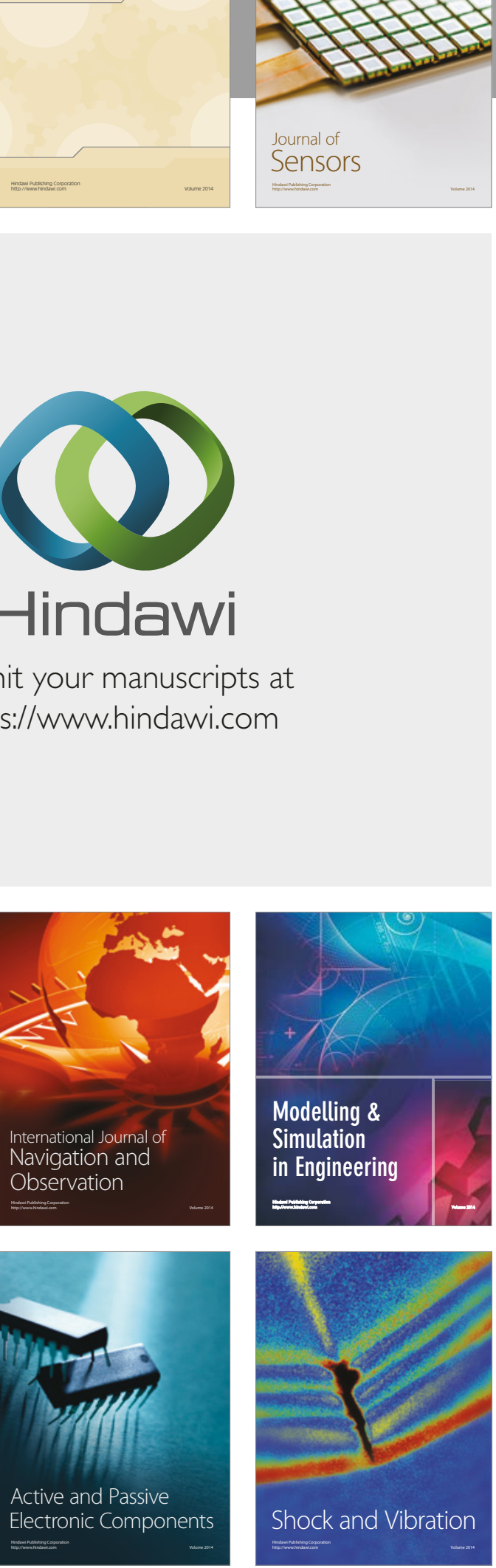
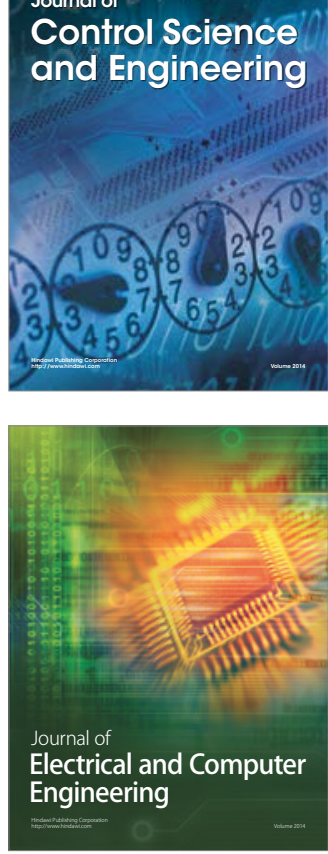

Distributed

Journal of

Control Science

and Engineering
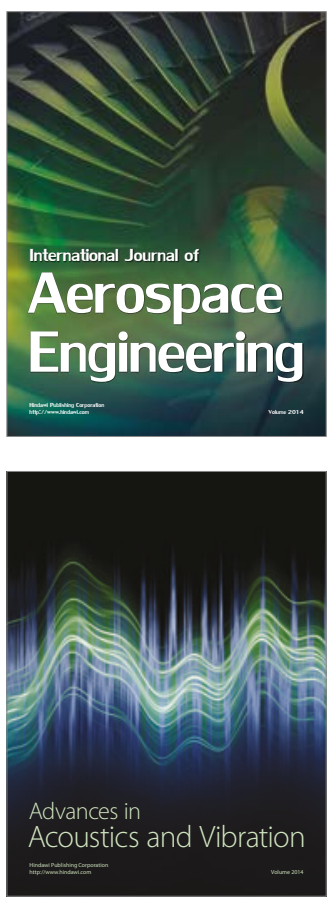

Sensor Networks 